\title{
Laboratory and space spectroscopy of $\mathrm{DCO}^{+}$
}

\author{
P. Caselli ${ }^{1}$ and L. Dore ${ }^{2}$ \\ 1 INAF - Osservatorio Astrofisico di Arcetri, Largo E. Fermi 5, 50125 Firenze, Italy \\ e-mail: caselli@arcetri.astro.it \\ 2 Dipartimento di Chimica "G. Ciamician", Università di Bologna, via Selmi 2, 40126 Bologna, Italy \\ e-mail: luca.dore@unibo.it
}

Received 5 October 2004 / Accepted 16 December 2004

\begin{abstract}
The rotational spectra of $\mathrm{DCO}^{+}$and its two isotopomers $\mathrm{D}^{13} \mathrm{CO}^{+}$and $\mathrm{DC}^{18} \mathrm{O}^{+}$, produced in a negative glow discharge cell, have been recorded in the 137-792 GHz region, which includes lines from $J=2 \leftarrow 1$ up to $J=11 \leftarrow 10$. The determined rotational and centrifugal distortion constants allow to predict the $\mathrm{DCO}^{+}$rotational spectrum up to $1000 \mathrm{GHz}$ with an accuracy of 1 part in $10^{8}$ or better. This is important for kinematic studies of dense molecular cloud cores and for future far-infrared observations. We also report on the first detection of the hyperfine structure of the $\mathrm{DCO}^{+}(1-0)$ line made at the IRAM $30 \mathrm{~m}$ antenna, toward the quiescent starless cloud core L1512, in the Taurus Molecular Cloud. We point out that this is the first observation of the hyperfine splitting due to the deuteron. This allowed us to quantify the effects of the hyperfine splitting on the line width determination; if the hyperfine structure is not taken into account in the line fit, the $\mathrm{DCO}^{+}(1-0)$ line width is overestimated by a significant factor $(\gtrsim 2)$.
\end{abstract}

Key words. molecular data - methods: laboratory - ISM: individual objects: L1512 - ISM: molecules - radio lines: ISM

\section{Introduction}

In the past few years it has been realized how important is to know with high precision the frequencies of the molecular transitions used to investigate the physics and the chemistry of interstellar clouds and star forming regions (e.g. Mardones et al. 1997; Lee et al. 1999, 2001). This is because spectral line observations are unique tools to study internal motions of dense molecular cloud material, within which stars will be or are forming. In this sense, molecular line observations are crucial to test current models of cloud core and star formation and give insights on molecular cloud evolution.

In particular, before low-mass stars form, the progenitor (or starless) core is typically a cold $(\sim 10 \mathrm{~K})$ and quiescent region, where the (optically thin) line widths are slightly (approximately a factor of 2) larger than the thermal value $\Delta v_{\mathrm{T}}$ (e.g. Caselli et al. 2002). This means that $\Delta v_{\mathrm{obs}} \simeq 2 \times \Delta v_{\mathrm{T}} \simeq 0.3$ $(T / 10 \mathrm{~K})^{1 / 2}\left(A_{\mathrm{X}} / 30 \mathrm{amu}\right)^{-1 / 2} \mathrm{~km} \mathrm{~s}^{-1}$, where $T$ is the gas temperature and $A_{\mathrm{X}}$ is the molecular weight. This already shows that to determine the LSR velocity of a cold $(T=10 \mathrm{~K})$ cloud with an accuracy comparable to the standard deviation of the corresponding Gaussian line profile $\left(\sigma_{\mathrm{V}_{\mathrm{LSR}}}=\Delta v_{\text {obs }} / \sqrt{8 \ln (2)} \lessgtr\right.$ $0.1 \mathrm{~km} \mathrm{~s}^{-1}$ ), for a molecular species with $A_{\mathrm{X}} \sim 30 \mathrm{amu}$ (such as $\mathrm{CO}, \mathrm{HCO}^{+}$, and $\mathrm{DCO}^{+}$), the observed line frequency should have an uncertainty of $\Delta v(\mathrm{kHz}) \lessgtr 0.1 \times 3.3 \times v_{0}(\mathrm{GHz})$ (where $v_{0}$ is the rest frequency), or about $30 \mathrm{kHz}$ at millimeter wavelengths.
If observed lines are optically thick and if they are tracing inward motions, they appear broader and asymmetric, typically double peaked, with the blue peak stronger than the red peak (e.g. Leung \& Brown 1977; Zhou et al. 1990). However, to quantify the infall velocity one needs to observe an optically thin tracer and compare the thin and thick profiles (e.g. Myers et al. 1996). For this purpose, one typically uses the normalized velocity difference between the thin and thick lines ( $\delta V$, as defined by Mardones et al. 1997). In this case, the condition $\delta V / \sigma_{\delta V}>3$ is only obtained if the error on $V$ is $\sigma_{V} \lesssim 0.01 \mathrm{~km} \mathrm{~s}^{-1}$ (assuming average values of $\delta V \sim 0.2$, $\sigma_{\Delta V} \sim 0.03 \mathrm{~km} \mathrm{~s}^{-1}$, and $\Delta V \simeq 0.3 \mathrm{~km} \mathrm{~s}^{-1}$; see Mardones et al. 1997). This implies a frequency precision of $\sigma_{v}(\mathrm{kHz}) \lesssim$ $0.03 \times v_{0}(\mathrm{GHz})$, or about $3 \mathrm{kHz}$ at $\sim 100 \mathrm{GHz}$. All this demonstrates the need of laboratory work to accurately measure line frequencies. Gottlieb et al. (2003) have also pointed out that it is a challenge to the spectroscopist the determination of line frequencies with a precision large enough to identify motions in molecular cloud cores from tiny velocity shifts, which typically require a precision of a few parts in $10^{8}$. In the case of $\mathrm{DCO}^{+}$ this is equivalent to $1.7 \%$ of the Doppler line width at $77 \mathrm{~K}$.

$\mathrm{DCO}^{+}$is a particularly suitable species to study the chemistry, in particular the deuterium fractionation (e.g. Saito et al. 2002) and the electron fraction (e.g. Guélin et al. 1977; Caselli et al. 1998; Williams et al. 1998), and the kinematics of the dense cores. The lower rotational transitions are easily detected, as it is proved by the large amount of literature on 
Table 1. Observed rotational transition of $\mathrm{DCO}^{+}(\mathrm{MHz})$.

\begin{tabular}{rrrrrrr}
\hline \hline$J^{\prime}$ & $J$ & Bogey et al. (1981) & Sastry et al. (1981) & Diff. $^{a} / \mathrm{ppb}$ & This work & Radiastronomy $^{b}$ \\
\hline 2 & 1 & 144077.342 & 144077.319 & 160 & 144077.2890 & 144077.321 \\
3 & 2 & 216112.701 & 216112.604 & 449 & 216112.5822 & \\
4 & 3 & 288144.018 & 288143.911 & 371 & 288143.8583 & 288143.911 \\
5 & 4 & 360169.980 & 360169.881 & 275 & 360169.7783 & 360169.830 \\
6 & 5 & & 432189.033 & & 432189.0052 & \\
\hline
\end{tabular}

${ }^{a}$ Difference between measurements of Bogey et al. (1981) and of Sastry et al. (1981).

${ }^{b}$ Lovas (2004).

this molecular ion, from interstellar clouds (e.g. Wootten et al. 1982) to dense cloud cores (e.g. Butner et al. 1995), to circumstellar disks (van Dishoeck et al. 2003). $\mathrm{DCO}^{+}$has been recently surveyed in a large sample of starless cores by Lee et al. (2004), with the aim of detecting infall motions. However, the previous measurements of $\mathrm{DCO}^{+}$millimeter-wave frequencies, carried out in two different laboratories (Bogey et al. 1981; Sastry et al. 1981), gave results differing up to 45 part in $10^{8}$ for the $J=3 \leftarrow 2$ line (see Table 1); therefore there is a need for more accurate frequency measurements of $\mathrm{DCO}^{+}$lines in order to use them in the study of star-forming molecular cloud cores. In this paper, we repeat with far better precision the old measurements of millimeter-wave transitions of $\mathrm{DCO}^{+}$, $\mathrm{D}^{13} \mathrm{CO}^{+}$, and $\mathrm{DC}^{18} \mathrm{O}^{+}$(Bogey at al. 1981), and we add new measurements of submillimeter-wave transitions: the spectroscopic constants derived from the analysis of the frequency data enable to predict spectra up to $1000 \mathrm{GHz}$ with an accuracy of at least 1 part in $10^{8}$ for $\mathrm{DCO}^{+}, 5$ parts in $10^{8}$ for $\mathrm{D}^{13} \mathrm{CO}^{+}$, and of 1 part in $10^{7}$ for $\mathrm{DC}^{18} \mathrm{O}^{+}$; all the isotopomers have been observed in natural abundance.

\section{Observations}

\subsection{Laboratory measurements}

The laboratory spectrum was observed with a frequencymodulated millimeter-wave spectrometer (Cazzoli \& Dore 1990a) equipped with a double-pass negative glow discharge cell (Dore et al. 1999) made of a Pyrex tube $3.25 \mathrm{~m}$ long and $5 \mathrm{~cm}$ in diameter. The radiation source was a frequency multiplier driven by a Gunn diode oscillator working in the region 68-76 GHz (Farran Technology Limited) for the lines up to $J=4 \leftarrow 3$; for the high frequency transitions, a doubler in cascade with a multiplier (RPG - Radiometer Physics GmbH) was driven by Gunn oscillators working in the region 75$115 \mathrm{GHz}$ (J. E. Carlstrom Co and RPG) to cover frequencies up to $792 \mathrm{GHz}$. Two phase-lock loops allow the stabilization of the Gunn oscillator with respect to a frequency synthesizer, which is driven by a $5-\mathrm{MHz}$ rubidium frequency standard. The frequency modulation of the radiation is obtained by sinewave modulating with low distortion (total harmonic distortion less than $0.01 \%$ ) the reference signal of the wide-band Gunnsynchronizer. The signal, detected by a liquid-helium-cooled InSb hot electron bolometer (QMC Instr. Ltd. type QFI/2), is demodulated at 2- $f$ by a lock-in amplifier.
$\mathrm{DCO}^{+}$was produced by flowing a $1: 1$ mixture of $\mathrm{CO}_{2}$ and $\mathrm{D}_{2}$ (1 mTorr) in Ar buffer gas with a total pressure of about 5 mTorr and discharging with a current of a few $\mathrm{mA} ; \mathrm{D}^{13} \mathrm{CO}^{+}$ and $\mathrm{DC}^{18} \mathrm{O}^{+}$were observed in natural abundance. The cell was cooled at $77 \mathrm{~K}$ by liquid nitrogen circulation, and an axial magnetic field up to about $400 \mathrm{G}$ was applied throughout the length of the discharge. With this longitudinal magnetic field applied, ions are produced and observed in the negative glow (De Lucia et al. 1982), which is a nearly field free region and where they are expected to show negligible Doppler shift due to the drift velocity, occurring, instead, in the positive column (Sastry et al. 1981) where a low axial electric field is present. In addition, the double-pass arrangement would compensate for such a shift, if present.

A typical spectrum is recorded by sweeping the frequency up and down (several times if signal averaging is needed) in steps of $5 \mathrm{kHz}$ at a rate of $0.8 \mathrm{MHz} \mathrm{s}^{-1}$, with a lock-in amplifier time constant of $3 \mathrm{~ms}$ and a frequency modulation depth comparable to the Doppler width or larger for the rarer isotopomers. Since we have full flexibility in controlling scanning rate, number of data points and modulation depth, the values of these parameters have been adjusted to prevent any bias of the measured transition frequency, which is recovered from a line shape analysis of the spectral profile (Cazzoli \& Dore 1990a; Dore 2003).

\section{2. $\mathrm{DCO}^{+}(1-0)$ toward the quiescent cloud core L1512}

The $\mathrm{DCO}^{+}(1-0)$ spectrum toward the quiescent Taurus starless core L1512 (Fig. 1) has been obtained with the IRAM$30 \mathrm{~m}$ antenna, located at Pico Veleta (Spain) in August 2004. The adopted coordinates were RA $(1950)=05^{\mathrm{h}} 00^{\mathrm{m}} 54.4^{\mathrm{s}}$, $\operatorname{Dec}(1950)=32^{\circ} 39^{\prime} 00.0^{\prime \prime}$. We used the recent extension of the $3 \mathrm{~mm}$ tuning range below $80 \mathrm{GHz}$ and carried out the observations using the frequency switching technique with a throw of $3.9 \mathrm{MHz}$. The spectral resolution is $3.3 \mathrm{kHz}$, which corresponds to $14 \mathrm{~m} / \mathrm{s}$ at $72 \mathrm{GHz}$. Given that below $\sim 75 \mathrm{GHz}$ the image gain depends strongly on the tuning parameters and often varies across the bandpass of the receiver, we measured the sideband gain ratio and corrected the observed spectrum accordingly. The pointing was checked every $2 \mathrm{~h}$ and found to be accurate within about $10^{\prime \prime}$ because of anomalous 


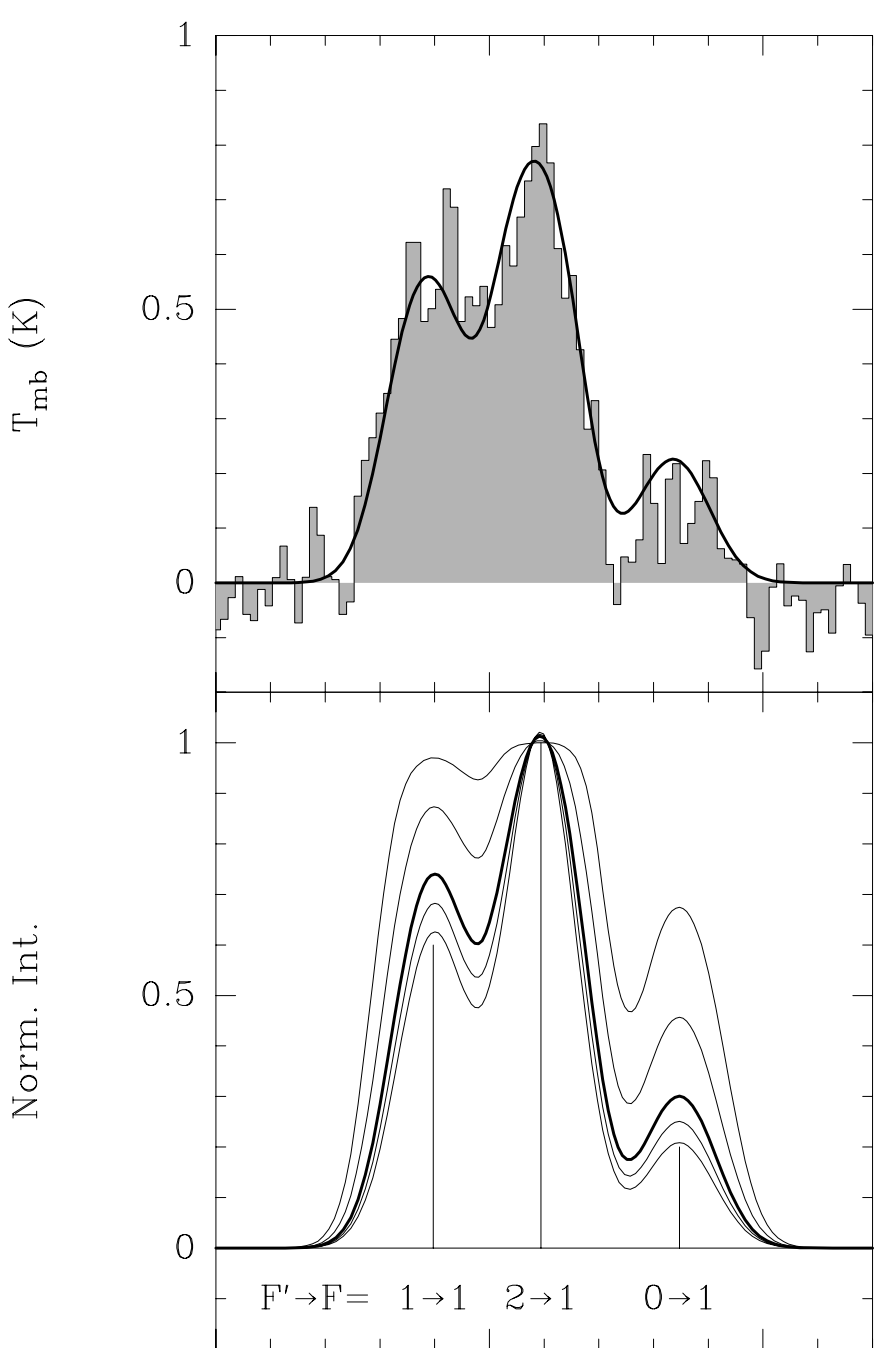

6.5 $\mathrm{V}_{\mathrm{LSR}}^{7}(\mathrm{~km} / \mathrm{s})$

Fig. 1. Top panel: observed spectrum of $\mathrm{DCO}^{+}(1-0)$ toward the quiescent starless cloud core L1512, in the Taurus Molecular Cloud. The units are in main beam brightness temperature, assuming a unity filling factor. This is the first spectrum showing the hyperfine structure of the line due to the deuteron electric quadrupole interaction. The line is significantly broadened by the hyperfine structure, and the width is a factor of 2.3 larger if the splitting is not taken into account in the fit. The black curve is the hfs fit performed in CLASS. Bottom panel: simulated spectrum of the $\mathrm{DCO}^{+}(1-0)$ line assuming the same parameters of the observed line ( $T_{\mathrm{ex}}=4 \mathrm{~K}$ and intrinsic $\left.\Delta v=0.15 \mathrm{~km} \mathrm{~s}^{-1}\right)$ but with different values of $\tau_{\text {TOT }}$ (from least to most intense curves: $0.1,1,2$ (as observed), 5, and 10. Vertical bars indicate the positions of the three hyperfines with their relative intensities in the optically thin limit.

refraction problems during very good atmospheric conditions. The half power beam width $(H P B W)$ was $33^{\prime \prime}$. The units are main beam brightness temperature, assuming a source filling factor of unity. The beam and forward efficiencies were 0.79 and 0.95 , respectively. The rms noise of the spectrum is $0.09 \mathrm{~K}$.

Figure 1 clearly shows that the $\mathrm{DCO}^{+}(1-0)$ line profile is complex and contains structure. Indeed, the three detected features are consistent with the hyperfine splitting due to the interaction between the molecular electric field gradient and the electric quadrupole moment of the deuteron ( $\operatorname{spin}=1$ ), which produces the line splitting into three components. The velocity shifts predicted by theory are larger than the observed separations between the three components by about $0.1 \mathrm{~km} \mathrm{~s}^{-1}$, suggesting that the quadrupole constant needs to be refined. Thus, we estimated the separations performing a Gaussian fit to the three components of the $\mathrm{DCO}^{+}(1-0)$ spectrum. This allowed us to derive the velocity separations of $-0.198 \pm 0.012 \mathrm{~km} \mathrm{~s}^{-1}$ and $0.256 \pm 0.017 \mathrm{~km} \mathrm{~s}^{-1}$ between the main $\left(F^{\prime}, F=2,1\right)$ component and the low $\left(F^{\prime}, F=1,1\right)$ and high $\left(F^{\prime}, F=0,1\right)$ velocity components, respectively. With this new values of the frequency shifts and the relative intensities of the three components $(3 / 9,5 / 9,1 / 9$ for the $(1,1),(2,1)$ and $(0,1)$ hyperfines, respectively) we then performed an hfs fit using the IRAM reduction package CLASS, shown in Fig. 1 (upper panel) by the thick curve. Forcing the resulting LSR velocity $\left(V_{\mathrm{LSR}}\right)$ to coincide with that measured with the high sensitivity $\mathrm{N}_{2} \mathrm{H}^{+}(1-$ 0) spectrum reported in Caselli et al. (1995), which has been corrected to account for the new $\mathrm{N}_{2} \mathrm{H}^{+}(1-0)$ frequency value $\left(93176.2608 \mathrm{MHz} \pm 6 \mathrm{kHz}\right.$ for the component $F_{1}, F=0,1 \rightarrow$ $1,2)$ estimated by Dore et al. (2004), we obtain the frequency values reported in Table 2 . These new frequencies have finally been used to refine the quadrupolar constant (see next section).

The hfs fit in CLASS gives the following parameters: $V_{\mathrm{LSR}}=7.094 \pm 0.004 \mathrm{~km} \mathrm{~s}^{-1}$, intrinsic (i.e. corrected for optical depth effects) line width $\Delta v=0.149 \pm 0.008 \mathrm{~km} \mathrm{~s}^{-1}$, total optical depth (i.e. the sum of the optical depths of the three hyperfines) $\tau_{\mathrm{TOT}}=1.9 \pm 0.5$, excitation temperature $T_{\mathrm{ex}}=4 \pm 1 \mathrm{~K}$. We note here that the observed line width is only 1.25 times larger than the $\mathrm{DCO}^{+}$thermal width $\Delta v_{\mathrm{T}}$, assuming a kinetic temperature of $10 \mathrm{~K}$ (or $\Delta v=\Delta v_{\mathrm{T}}$ if $T=15 \mathrm{~K}$ ). If the hyperfine structure is not taken into account, the $\mathrm{DCO}^{+}(1-0)$ line width obtained with a simple Gaussian fit is a factor of 2.3 times larger, in net contrast with the narrow $\left(\sim 0.18 \mathrm{~km} \mathrm{~s}^{-1}\right)$ $\mathrm{N}_{2} \mathrm{H}^{+}(1-0)$ line width observed in the same position (Caselli et al. 1995). Therefore, we conclude that the differences in the widths of $\mathrm{DCO}^{+}(1-0)$ and $\mathrm{NH}_{3}(1,1)$ lines observed in previous work (e.g. compare Butner et al. 1995; with Benson \& Myers 1989) are likely due to neglecting the hyperfine splitting. The bottom panel of Fig. 1 shows how the line profile changes in case of large optical depths. For $\tau_{\mathrm{TOT}}=10$, the weakest $\left(F^{\prime}\right.$, $F=0,1)$ component becomes bright enough to further enlarge the line width by other $\sim 0.25 \mathrm{~km} \mathrm{~s}^{-1}$ (so that the total linewidth will be about $0.6 \mathrm{~km} \mathrm{~s}^{-1}$, if the splitting is not considered in the fit).

The effects of the hyperfine structure on the line width and profile become less important for higher $J$ transitions. In Fig. 2, the simulated spectrum of the $\mathrm{DCO}^{+}(2-1)$ line, with the same parameters as the $\mathrm{DCO}^{+}(1-0)$ line in Fig. 1, is shown. The six hyperfine components are blended together, but there is still some $(\sim 20 \%)$ extra-broadening and a small $\left(0.01 \mathrm{~km} \mathrm{~s}^{-1}\right)$ line center shift resulting from the hfs splitting, which should be taken into account when analysing $\mathrm{DCO}^{+}(2-1)$ profiles. In particular, this may be important to quantify the infall velocity in the central regions of starless cores, as recently done by Lee et al. (2004). On the other hand, the components of the $J=3 \rightarrow 2$ transition (as well as those of higher $J$ transitions) 
Table 2. Rotational transition frequencies and spectroscopic constants of $\mathrm{DCO}^{+}$.

\begin{tabular}{|c|c|c|c|c|c|}
\hline & $\left(F^{\prime}\right)$ & $J(F)$ & Observed/MHz & Obs.-calc./kHz & Uncertainties $/ \mathrm{kHz}^{a}$ \\
\hline & (0) & $0(1)$ & 72039.2413 & -0.1 & 7.3 \\
\hline & (2) & $0(1)$ & 72039.3028 & -0.3 & 6.1 \\
\hline & (1) & $0(1)$ & 72039.3504 & -0.2 & 6.7 \\
\hline 2 & & 1 & $144077.2890^{b}$ & 3.9 & 5 \\
\hline 3 & & 2 & 216112.5822 & 2.1 & 5 \\
\hline 4 & & 3 & 288143.8583 & 0.5 & 5 \\
\hline 5 & & 4 & 360169.7783 & -1.2 & 5 \\
\hline 6 & & 5 & 432189.0052 & -1.3 & 5 \\
\hline 7 & & 6 & 504200.1999 & -0.5 & 5 \\
\hline 8 & & 7 & 576202.0239 & 1.1 & 5 \\
\hline 9 & & 8 & 648193.1357 & 0.0 & 5 \\
\hline 10 & & 9 & 720172.2024 & 1.1 & 10 \\
\hline 11 & & 10 & 792137.8811 & -1.2 & 10 \\
\hline \multicolumn{6}{|c|}{$\sigma_{\text {fit }}^{c} / \mathrm{kHz} 1.3$} \\
\hline \multicolumn{3}{|c|}{ Constant $^{d}$} & This work & Previous $^{e}$ & $\mathrm{DC}^{15} \mathrm{~N}$ \\
\hline \multirow{5}{*}{\multicolumn{3}{|c|}{$\begin{array}{l}B / \mathrm{MHz} \text { rotational } \\
D_{J} / \mathrm{kHz} \text { quartic c. d. } \\
H_{J} / \mathrm{Hz} \text { sextic c. d. } \\
e q Q / \mathrm{kHz} \text { quadrupole } \\
C_{I} / \mathrm{kHz} \text { spin rotation }\end{array}$}} & $36019.76765(14)$ & $36019.784(2)$ & \\
\hline & & & $55.7960(22)$ & $55.71(12)$ & $54.3924(15)^{g}$ \\
\hline & & & $0.054(11)$ & & $0.0633(12)^{g}$ \\
\hline & & & $147.8(35)$ & & $200.6(5)^{h}$ \\
\hline & & & $-1.59(78)$ & & $-1.9(1)^{h}$ \\
\hline \multicolumn{6}{|c|}{ Uncertainties estimated as explained in the text. } \\
\hline \multicolumn{6}{|c|}{ Not included in the fit, see text. } \\
\hline \multicolumn{6}{|c|}{$\left(\Sigma(\text { obs.-calc. })^{2} / \text { degrees of freedom }\right)^{1 / 2}$} \\
\hline \multicolumn{6}{|c|}{ Standard errors are reported in parentheses in units of the last quoted digits. } \\
\hline \multicolumn{6}{|c|}{ Bogey et al. (1981). } \\
\hline \multicolumn{6}{|c|}{ Centrifugal distortion. } \\
\hline \multicolumn{6}{|c|}{ Brünken et al. (2004). } \\
\hline \multicolumn{6}{|c|}{ Cazzoli \& Dore (1990b). } \\
\hline
\end{tabular}

are heavily blended together, and the line width measurement is not affected at all by their presence.

\section{Analysis and discussion}

The measured transition frequencies of $\mathrm{DCO}^{+}, \mathrm{D}^{13} \mathrm{CO}^{+}$, and $\mathrm{DC}^{18} \mathrm{O}^{+}$are listed in Tables 2-4; they are mean values obtained from 5 to 14 measurements. The standard errors of the mean result unrealistically small (for $\mathrm{DCO}^{+}$they range from 0.1 to $1 \mathrm{kHz}$ ), therefore the uncertainties reported in the last column of each table were estimated from the ratio of linewidth to signal-to-noise ratio, and were roughly scaled according to the standard errors.

The experimental data were fitted, in a weighted leastsquares procedure, to the standard expression for the frequency of the rotational transition $J+1 \leftarrow J$ :

$v_{0}=2 B(J+1)-4 D_{J}(J+1)^{3}+H_{J}(J+1)^{3}\left[(J+1)^{3}-J^{3}\right]$,

where $B$ is the rotational constant and $D_{J}$ and $H_{J}$ are the quartic and sextic centrifugal distortion constants, respectively; the weights were the inverse-square of the uncertainties. The standard deviation $\sigma_{\text {fit }}$ of the fit for each isotopomer is just a few $\mathrm{kHz}$ with the inclusion of $H_{J}$ as fit parameter. An assessment of the significance of the latter can be carried out by comparison with DCN centrifugal distortion constants: the values (Brünken et al. 2004) reported in Tables 2 and 3 result comparable with the values of $D_{J}$ and $H_{J}$ derived in this work for $\mathrm{DCO}^{+}$and $\mathrm{D}^{13} \mathrm{CO}^{+}$, therefore these constants may be assumed correctly determined.

In the case of $\mathrm{DCO}^{+}$, the three hyperfine frequencies of the $J=1 \rightarrow 0$ transition, accurately determined as reported above, could be used to derive the quadrupole coupling (eqQ) and spin rotation $\left(C_{I}\right)$ constants of the $\mathrm{D}$ nucleus (spin quantum number $I=1)$ according to the expression:

$$
\begin{array}{r}
v_{h f}=v_{0}-e q Q\left[Y\left(J+1, I, F^{\prime}\right)-Y(J, I, F)\right] \\
+C_{I}\left[C\left(J+1, I, F^{\prime}\right)-C(J, I, F)\right],
\end{array}
$$

where $F$ is the total angular momentum $(\boldsymbol{F}=\boldsymbol{J}+\boldsymbol{I})$ quantum number, $C(J, I, F)=F(F+1)-I(I+1)-J(J+1)$ and the Casimir function $Y(J, I, F)$ is given by:

$Y(J, I, F)]=\frac{\frac{3}{4} C(C+1)-I(I+1) J(J+1)}{2(2 I-1)(2 J+3) I(2 I-1)}$.

The Pickett's SPFIT fitting program (Pickett 1991) allowed us to carry out simultaneously the hfs and centrifugal analyses in 


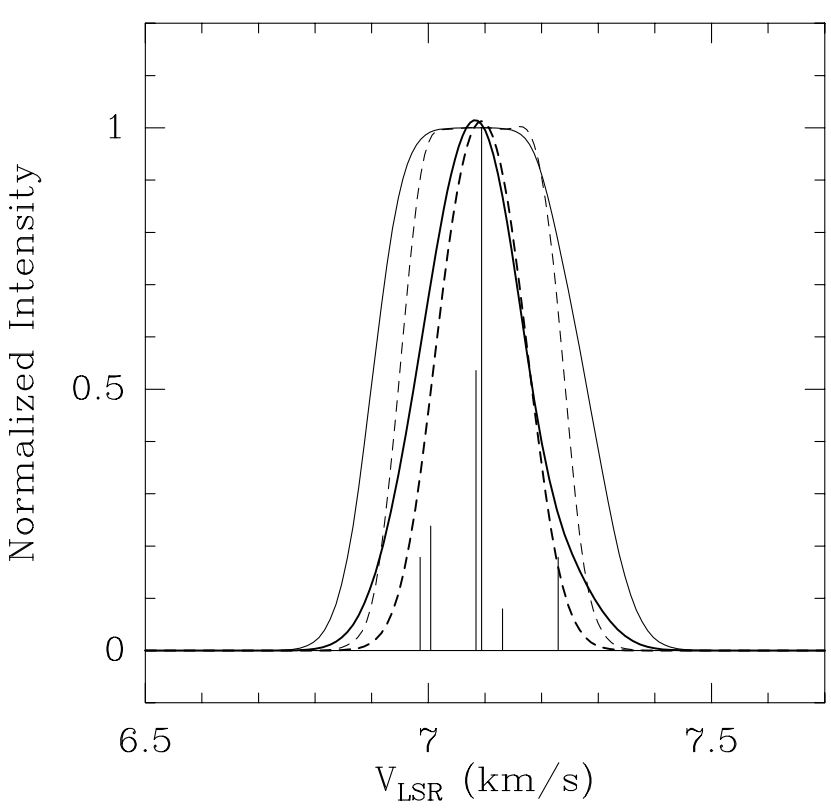

Fig. 2. Simulated spectrum of the $J=2 \rightarrow 1$ transition of $\mathrm{DCO}^{+}$in L1512, including the hyperfine splitting due to the deuteron electric quadrupole interaction (black curves). The assumed excitation temperature is $4 \mathrm{~K}$ and the intrinsic linewidth is $0.15 \mathrm{~km} \mathrm{~s}^{-1}$, as found for the $\mathrm{DCO}^{+}(1-0)$ line, whereas the total optical depth is 1 (thick curve) and 10 (thin curve). Dashed curves are the expected $\mathrm{DCO}^{+}(2-$ 1) lines in case of no hyperfine structure, again assuming $\tau_{\mathrm{TOT}}=1$ (thick dashed curve) and 10 (thin dashed curve). Vertical lines mark the velocities of the 6 hyperfine components with relative intensities given by their statistical weight. The hyperfine splitting leads to a $\sim 20$ and $\sim 30 \%$ increase of the line width for $\tau_{\text {TOT }}=1$ and 10 , respectively.

a global fit of laboratory and radioastronomical transition frequencies, whose weights were the inverse-square of the uncertainties. For the hyperfines, their assumed uncertainties account for both the standard errors of the hfs fit and the $6 \mathrm{kHz}$ uncertainty of the $\mathrm{N}_{2} \mathrm{H}^{+}(1-0)$ reference line (Dore et al. 2004).

Tables 2-4 list, for each isotopomer, the experimental frequencies with their uncertainties and the residuals of the leastsquares fit carried out to determine the spectroscopic constants reported at the bottom of the table. These accurate constants (see next paragraphs for a discussion of accuracy) allow us to recommend the rest frequencies reported in Table 5: the hyperfine components of the $J=1 \leftarrow 0$ and $J=2 \leftarrow 1$ transitions are listed for $\mathrm{DCO}^{+}$, while unsplit transition frequencies from $J=2$ up to $J=14$ are reported for all three isotopomers. It is worth mentioning that the quoted uncertainties in Table 5 are $1 \sigma$ errors derived from the least-squares variance-covariance matrix for the fitted spectroscopic parameters.

As for the accuracy of the measured transition frequencies, the sources of systematic error are of two types: those due to the experimental set-up and procedures, and those related to the physics of molecules in the experimental environment.

The sources of the first kind include: accuracy of the frequency standard, harmonic distortion of the frequency modulation, integration due to slow lock-in time constant, asymmetry in the line shape due to etalon effect in the absorption cell.

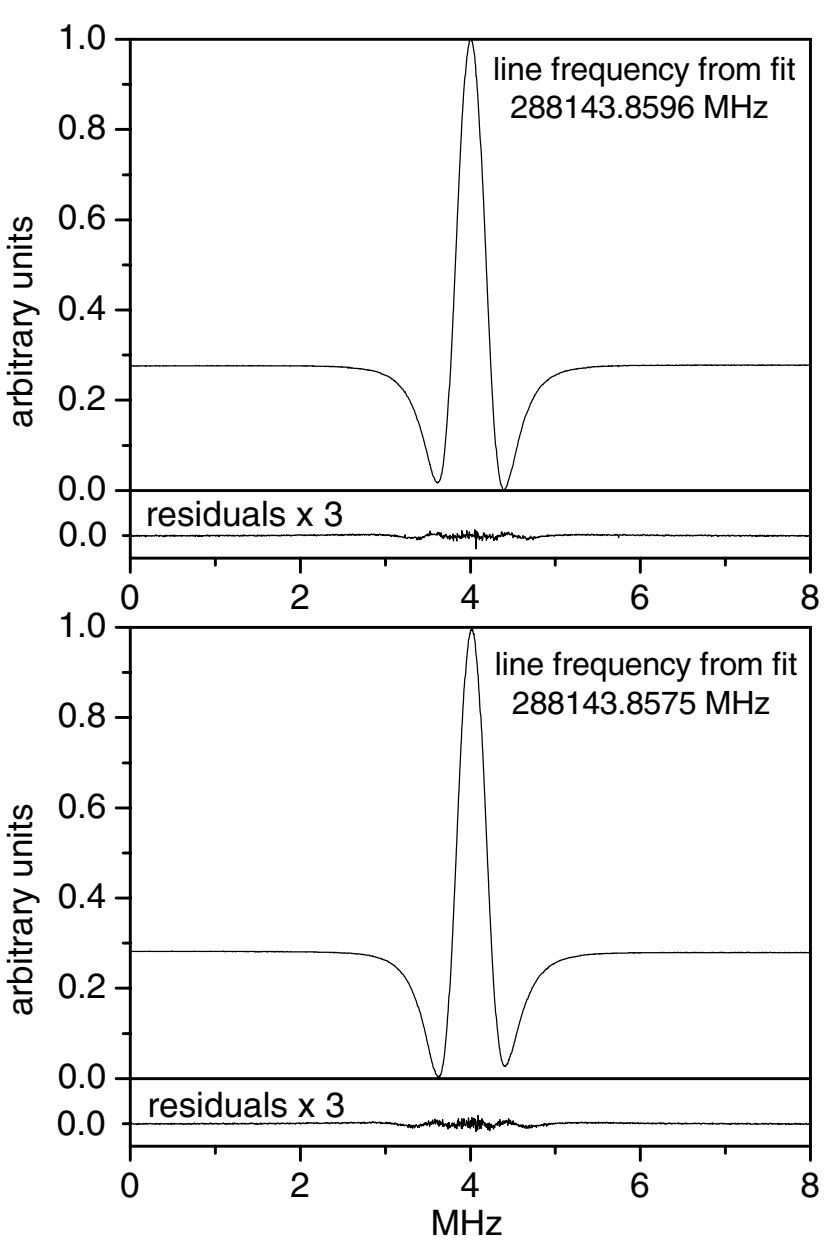

Fig. 3. Spectra of the $J=4 \leftarrow 3$ transition of $\mathrm{DCO}^{+}$with opposite line asymmetry due to different standing wave pattern in the cell: the 1601 points of each spectrum were recorded for a total time of $39 \mathrm{~s}$ at a rate of $0.8 \mathrm{MHz} \mathrm{s}^{-1}$ with a lock-in time constant of $1 \mathrm{~ms}$. Their profile has been fit to a model Galatry profile with the inclusion of a dispersion term to recover the line frequency.

The well tested performances of the spectrometer (Cazzoli \& Dore 1990a; Puzzarini et al. 2002) and a judicious choice of the lock-in time constant (less than 1/100 of the rising time during the line recording) allowed all these error sources, except the last, to be rendered negligible: Fig. 3 shows, in fact, a pair of spectra of the $J=5 \leftarrow 4$ transition of $\mathrm{DCO}^{+}$with opposite asymmetry. The shift of the apparent center frequency, however, may be accounted for by a full profile analysis with a polynomial function describing the baseline and a dispersion term included; this procedure gives an accurate line center (Dore 2003).

The second kind of error in the line center includes: Doppler shift due to ion drift in the discharge, gas flow through the cell due to pumping, pressure shift. The first two causes of line shift should be negligible in the present experimental conditions, and are, anyway, largely suppressed by the double-pass arrangement. As for the frequency shift by Ar pressure (about 4 mTorr in this experiment), Buffa et al. (1994) have shown in the case of $\mathrm{HCO}^{+}$that it is most significant for the $J=1 \leftarrow 0$ 
Table 3. Rotational transition frequencies and spectroscopic constants of $\mathrm{D}^{13} \mathrm{CO}^{+}$.

\begin{tabular}{|c|c|c|c|c|}
\hline$J^{\prime}$ & $J$ & Observed/MHz & Obs.-calc./kHz & Uncertainties $/ \mathrm{kHz}^{a}$ \\
\hline 2 & 1 & $141465.1331^{b}$ & 3.4 & 5 \\
\hline 3 & 2 & 212194.4920 & 1.9 & 5 \\
\hline 4 & 3 & 282920.0055 & 0.3 & 5 \\
\hline 5 & 4 & 353640.3923 & -1.3 & 5 \\
\hline 6 & 5 & 424354.3718 & -1.8 & 5 \\
\hline 7 & 6 & 495060.6646 & 0.4 & 5 \\
\hline 8 & 7 & 565757.9861 & 2.0 & 5 \\
\hline 9 & 8 & 636445.0518 & -0.8 & 5 \\
\hline 10 & 9 & 707120.5885 & -0.7 & 15 \\
\hline 11 & 10 & 777783.3159 & 2.3 & 50 \\
\hline \multicolumn{5}{|c|}{$\sigma_{\text {fit }}^{c} / \mathrm{kHz} 1.8$} \\
\hline & & This work & Previous $^{e}$ & $\mathrm{D}^{13} \mathrm{C}^{15} \mathrm{~N}^{f}$ \\
\hline & ational & $35366.70968(21)$ & $35366.712(11)$ & \\
\hline & $\operatorname{artic~c.~d.~}{ }^{g}$ & $53.4083(40)$ & $53.20(53)$ & $52.26535(96)$ \\
\hline & tic c. d..$^{g}$ & $0.048(24)$ & & $0.06074(73)$ \\
\hline $\begin{array}{l}\text { Un } \\
\text { No } \\
(\Sigma( \\
\text { Sta } \\
\mathrm{Bo} \\
\mathrm{Bri} \\
\mathrm{Ce}\end{array}$ & $\begin{array}{l}\text { ties estimate } \\
\text { led in the fit } \\
\text { lc. })^{2} / \text { degrees } \\
\text { rrors are rep } \\
\text { l. (1981). } \\
\text { t al. (2004). } \\
\text { l distortion. }\end{array}$ & $\begin{array}{l}\text { as explained in the } t \\
\text { see text. } \\
\text { of freedom })^{1 / 2} \text {. } \\
\text { orted in parentheses }\end{array}$ & units of the last qu & oted digits. \\
\hline
\end{tabular}

transition; a semiclassical estimate for $\mathrm{DCO}^{+}$broadened by $\mathrm{Ar}$ (Buffa 2004) indicates that, among the rotational lines considered here, only the $J=2 \leftarrow 1$ center frequency should be slightly shifted (about $3 \mathrm{kHz}$ ); therefore it has been excluded from the fit.

In the present case, there are also the hyperfine contributions of the $\mathrm{D}$ and ${ }^{13} \mathrm{C}$ nuclei which may affect the accuracy of the determined transition frequencies. These effects are more significant for the lower $J$ transitions, therefore the most affected among those included in the fit should be the $J=3 \leftarrow 2$. Its hf structure was predicted assuming the hf constants from the present work for D and from Schmid-Burgk et al. (2004) for ${ }^{13} \mathrm{C}$; then, to simulate the blended experimental profile, modulated Voigt functions (see Dore 2003) were summed at each hyperfine frequency with the relative intensity as weighting factor; finally, the center frequency of the synthetic profile was measured to check for a shift with respect to the assumed unperturbed $J=3 \leftarrow 2$ transition frequency. From this procedure carried out for all three isotopomers, it turned out that the apparent line center is shifted at most by $0.3 \mathrm{kHz}$ from the unperturbed center frequency: this indicates that the hyperfine structure is irrelevant in this case, and a fortiori for the higher $J$ transitions, as seen in Sect. 2.2.

\section{Conclusions}

The present paper has shown how radioastronomical observations together with laboratory work are needed to determine with high precision the spectroscopic parameters of molecular species. In the laboratory, it has been possible to measure the frequencies of $\mathrm{DCO}^{+}, \mathrm{D}^{13} \mathrm{CO}^{+}$, and $\mathrm{DC}^{18} \mathrm{O}^{+}$lines up to $792 \mathrm{GHz}$ with a fairly high accuracy. New values of the rotational, quartic, and sextic distortion constants have been determined for the three isotopomers with such a precision to allow to predict spectra up to $1000 \mathrm{GHz}$ with an accuracy of at least 1 part in $10^{8}$ for $\mathrm{DCO}^{+}, 5$ parts in $10^{8}$ for $\mathrm{D}^{13} \mathrm{CO}^{+}$, and of 1 part in $10^{7}$ for $\mathrm{DC}^{18} \mathrm{O}^{+}$. Previous frequency estimates were much less accurate and did not allow to make accurate kinematic studies in dense molecular cloud cores.

Using the IRAM-30 m antenna, we detected for the first time the hyperfine structure of the $\mathrm{DCO}^{+}(1-0)$ line toward the quiescent starless core L1512, in Taurus. This is the first time that the hyperfine structure due to a deuteron has ever been observed; this gave us the possibility of obtaining the hyperfine parameters of $\mathrm{DCO}^{+}$. The $\mathrm{DCO}^{+}(1-0)$ frequency has also been estimated comparing the observed line with a high sensitivity spectrum of $\mathrm{N}_{2} \mathrm{H}^{+}(1-0)$, and using the new value of the $\mathrm{N}_{2} \mathrm{H}^{+}(1-0)$ line as calculated by Dore et al. (2004). This value is however still uncertain by $6 \mathrm{kHz}$ because of astronomical errors, and new sub-Doppler laboratory work on $\mathrm{N}_{2} \mathrm{H}^{+}$ is planned to improve this precision. The estimated $\mathrm{DCO}^{+}(1-$ 0 ) hf frequencies were included with their uncertainties in a weighted least-squares fit of the laboratory data to derive accurate spectroscopic constants. From the fitted constants, the hyperfines have been reestimated with an accuracy of better than 2.5 parts in $10^{8}$. 
Table 4. Rotational transition frequencies and spectroscopic constants of $\mathrm{DC}^{18} \mathrm{O}^{+}$.

\begin{tabular}{llccc}
\hline \hline$J^{\prime}$ & $J$ & Observed/MHz & Obs.-calc./kHz & Uncertainties $/ \mathrm{kHz}^{a}$ \\
\hline 2 & 1 & $137653.5239^{b}$ & 3.1 & 10 \\
3 & 2 & 206477.2402 & -0.9 & 5 \\
4 & 3 & 275297.3150 & 1.5 & 5 \\
5 & 4 & 344112.5213 & -1.1 & 5 \\
6 & 5 & 412921.6530 & 0.4 & 5 \\
7 & 6 & 481723.4893 & 0.3 & 5 \\
8 & 7 & 550516.8156 & -1.7 & 10 \\
9 & 8 & 619300.4281 & 4.4 & 35 \\
10 & 9 & 688073.0967 & 1.9 & 45 \\
$\sigma_{\text {fit }}^{c} / \mathrm{kHz} 2.5$ & & &
\end{tabular}

\begin{tabular}{lcc}
\hline Constant $^{d}$ & This work & Previous $^{e}$ \\
\hline$B / \mathrm{MHz}$ rotational & $34413.78556(18)$ & $34413.798(1)$ \\
$D_{J} / \mathrm{kHz}$ quartic c. d. & $50.6704(44)$ & $50.29(4)$ \\
$H_{J} / \mathrm{Hz}$ sextic c. d. ${ }^{f}$ & $0.109(34)$ & \\
\hline
\end{tabular}

${ }^{a}$ Uncertainties estimated as explained in the text.

${ }^{b}$ Not included in the fit, see text.

c $(\Sigma \text { (obs.-calc. })^{2} /$ degrees of freedom $)^{1 / 2}$.

${ }^{d}$ Standard errors are reported in parentheses in units of the last quoted digits.

$e$ Bogey et al. (1981).

${ }^{f}$ Centrifugal distortion.

Table 5. Recommended line frequencies ${ }^{a}(\mathrm{MHz})$ of $\mathrm{DCO}^{+}, \mathrm{D}^{13} \mathrm{CO}^{+}$, and $\mathrm{DC}^{18} \mathrm{O}^{+}$.

\begin{tabular}{llccr}
\hline \hline$J^{\prime}\left(F^{\prime}\right)$ & $J(F)$ & $\mathrm{DCO}^{+}$ & $\mathrm{D}^{13} \mathrm{CO}^{+}$ & $\mathrm{DC}^{18} \mathrm{O}^{+}$ \\
\hline $1(0)$ & $0(1)$ & $72039.2414(18)$ & & \\
$1(2)$ & $0(1)$ & $72039.3031(9)$ & & \\
$1(1)$ & $0(1)$ & $72039.3506(14)$ & & \\
$2(1)$ & $1(1)$ & $144077.2144(18)$ & & \\
$2(1)$ & $1(2)$ & $144077.2619(29)$ & & \\
$2(3)$ & $1(2)$ & $144077.2804(10)$ & & \\
$2(2)$ & $1(1)$ & $144077.2851(5)$ & & \\
$2(1)$ & $1(0)$ & $144077.3237(15)$ & & $206477.2411(7)$ \\
$2(2)$ & $1(2)$ & $144077.3326(23)$ & & $344112.5224(6)$ \\
3 & 2 & $216112.5800(6)$ & $212194.4901(9)$ & $412921.6526(6)$ \\
4 & 3 & $288143.8577(6)$ & $282920.0052(9)$ & $481723.4890(8)$ \\
5 & 4 & $360169.7795(6)$ & $353640.3936(8)$ & $550516.8173(13)$ \\
6 & 5 & $432189.0066(5)$ & $424354.3736(7)$ & $619300.4237(31)$ \\
7 & 6 & $504200.2004(6)$ & $495060.6642(8)$ & \\
8 & 7 & $576202.0228(7)$ & $565757.9841(9)$ & \\
9 & 8 & $648193.1356(8)$ & $636445.0526(11)$ & \\
10 & 9 & $720172.2013(11)$ & $707120.5892(28)$ & $688073.0947(72)$ \\
11 & 10 & $792137.8823(22)$ & $777783.3135(67)$ & $756833.618(14)$ \\
12 & 11 & $864088.8419(47)$ & $848431.946(13)$ & $825580.782(26)$ \\
13 & 12 & $936023.7433(88)$ & $919065.206(24)$ & $894313.375(43)$ \\
14 & 13 & $1007941.251(15)$ & $989681.816(40)$ & $963030.188(68)$ \\
15 & 14 & $1079840.028(24)$ & $1060280.497(62)$ & $1031730.011(103)$ \\
\hline
\end{tabular}

a The $1 \sigma$ uncertainties are reported in parentheses in units of the last quoted digits. 
The hyperfine structure of the $\mathrm{DCO}^{+}(1-0)$ line needs to be taken into account in the analysis of astronomical data, to avoid overestimates of the line width by more than a factor of 2 and for a correct interpretation of the line profile.

Acknowledgements. This work was supported by the Italian Ministry of Public Instruction, University and Research and by ASI (contract $\mathrm{I} / \mathrm{R} / 044 / 02$ ). It is a pleasure to thank Nuria Marcelino from IRAM, who kindly helped us during observations, and the referee, Mario Tafalla, for making useful comments which helped to clarify some aspects of the paper. We also thank Malcolm Walmsley for his critical reading of the manuscript.

\section{References}

Bogey, M., Demuynck, C., \& Destombes, J. L. 1981, Mol. Phys., 43, 1043

Brünken, S., Fuchs, U., Lewen, F., et al. 2004, J. Mol. Spectrosc., 225, 152

Buffa, G., Tarrini, O., Cazzoli, G., \& Dore, L. 1994, Phys. Rev. A, 49, 3557

Buffa, G. 2004, private communication

Butner, H. M., Lada, E. A., \& Loren, R. B. 1995, ApJ, 448, 207

Caselli, P., Myers, P. C., \& Thaddeus, P. 1995, ApJ, 455, L77

Caselli, P., Walmsley, C. M., Terzieva, R., \& Herbst, E. 1998, ApJ, 499, 234

Caselli, P., Benson, P. J., Myers, P. C., \& Tafalla, M. 2002, ApJ, 572, 238

Cazzoli, G., \& Dore, L. 1990a, J. Mol. Spectrosc., 141, 49

Cazzoli, G., \& Dore, L. 1990b, J. Mol. Spectrosc., 143, 231
De Lucia, F. C., Herbst, E., Plummer, G. M., \& Blake, G. A. 1982, J. Chem. Phys., 78, 2312

Dore, L., Degli Esposti, C., Mazzavillani, A., \& Cazzoli, G. 1999, Chem. Phys. Lett., 300, 489

Dore, L. 2003, J. Mol. Spect., 221, 93

Dore, L., Caselli, P., Beninati, S., et al. 2004, A\&A, 413, 1177

Gottlieb, C. A., Myers, P. C., \& Thaddeus, P. 2003, ApJ, 588, 655

Guelin, M., Langer, W. D., Snell, R. L., \& Wootten, H. A. 1977, ApJ, 217, L165

Lee, C. W., Myers, P. C., \& Tafalla, M. 1999, ApJ, 526, 788

Lee, C. W., Myers, P. C., \& Tafalla, M. 2001, ApJS, 136, 703

Lee, C. W., Myers, P. C., \& Plume, R. 2004, ApJS, 153, 523

Leung, C. M., \& Brown, R. L. 1977, ApJ, 214, L73

Lovas, F. J. 2004, J. Phys. Chem. Ref. Data, 33, 177

Mardones, D., Myers, P. C., Tafalla, M., et al. 1997, ApJ, 489, 719

Myers, P. C., Mardones, D., Tafalla, M., Williams, J. P., \& Wilner, D. J. 1996, ApJ, 465, L133

Pickett H. M. 1991 J. Mol. Spectrosc., 148, 371

Puzzarini, C., Cazzoli, G., \& Dore, L. 2002, J. Mol. Spectrosc., 216, 428

Saito, S., Aikawa, Y., Herbst, E., et al. 2002, ApJ, 569, 836

Sastry ,K. V. L. N., Herbst, E., \& De Lucia, F. C. 1981, J. Chem. Phys., 75,4169

Schmid-Burgk, J., Muders, D., Müller, H. S. P., \& Brupbacher-Gatehouse, B. 2004, A\&A, 419, 949

van Dishoeck, E. F., Thi, W.-F., \& van Zadelhoff, G.-J. 2003, A\&A, 400, L1

Williams, J. P., Bergin, E. A., Caselli, P., Myers, P. C., \& Plume, R. 1998, ApJ, 503, 689

Wootten, A., Loren, R. B., \& Snell, R. L. 1982, ApJ, 255, 160

Zhou, S., Evans, Neal J., II, Butner, H. M., et al. 1990, ApJ, 363, 168 